\title{
Approved Protocol
}

National Cancer Institute

\section{Source}

National Cancer Institute. Approved Protocol. NCI Thesaurus. Code C70745.

Protocol is approved by the review board. 